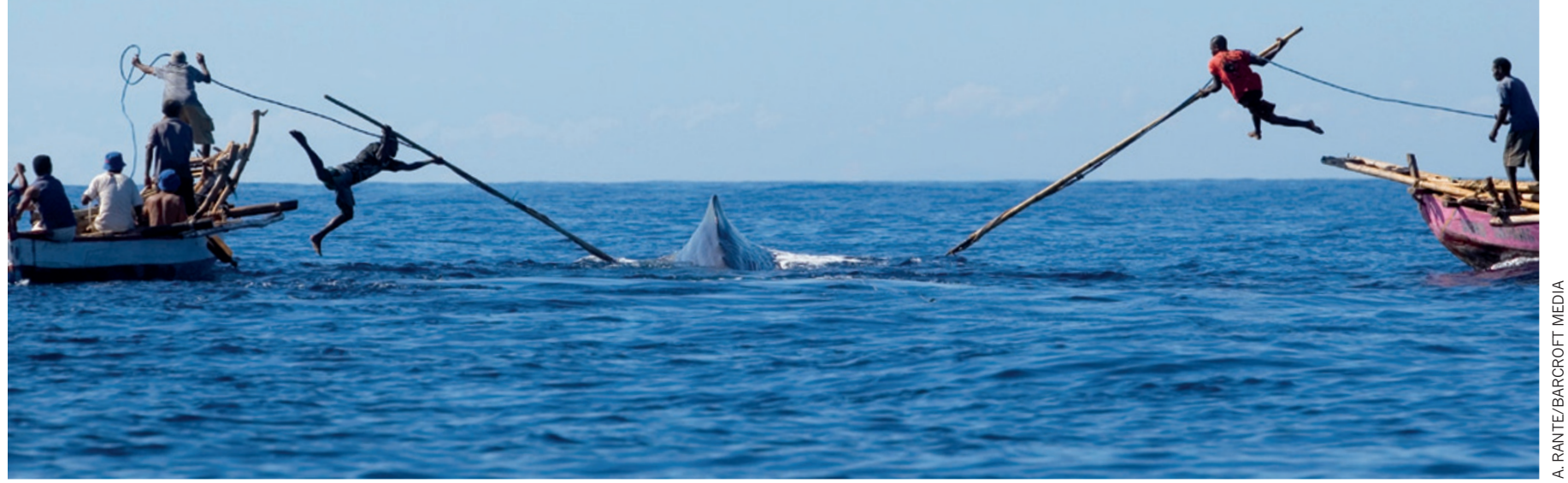

Showing high levels of cooperation, whale hunters from Lamalera in Indonesia work together to spear a whale.

EVOLUTION

\title{
Not so selfish
}

\section{A prescription for how human cooperation evolved will provoke much-needed debate about the origins of society, finds Peter Richerson.}

$\mathrm{H}$ umans are capable of remarkable feats of cooperation. Warfare is an extreme example: when under attack, hundreds or even millions of people might join forces to provide a mutual defence. In A Cooperative Species, economists Samuel Bowles and Herbert Gintis update their ideas on the evolutionary origins of altruism. Containing new data and analysis, their book is a sustained and detailed argument for how genes and culture have together shaped our ability to cooperate.

Modern hunting and gathering societies offer clues as to how human cooperation evolved. They are typically organized into tribes of a few hundred to a few thousand people. Each tribe is composed of smaller bands of around 75 individuals united by bonds of kinship and friendship. Formalized leadership is often weak, but cooperation is buttressed by social norms and institutions, such as marriage, kinship and property rights. The tribal scale of social organization probably evolved by the late Pleistocene (126,00011,700 years ago), or perhaps much earlier.

Human societies are diverse and competitive, often violently so. Charles Darwin conjectured in The Descent of Man (John Murray, 1871) that the main evolutionary motor behind human cooperation was intertribal competition, and suggested that cooperation evolved in two stages. In 'primeval' times, well before the dawn of recorded history, our ancestors came under selection for cooperative instincts, such as sympathy and group loyalty. In more recent 'civilized' times, laws and customs have fostered cooperation on ever larger scales. Darwin contended that the primeval social emotions, more than natural selection, drove the evolution of civilization.

Around 1900, concern with the Darwinian mechanisms of evolution died out in the emerging social sciences, shortly before the topic exploded in biology. Sociobiologist E. O. Wilson and others resurrected interest in the 1970s.

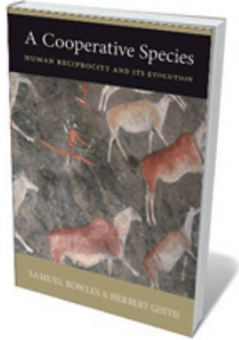

A Cooperative Species: Human Reciprocity and Its Evolution

SAMUEL BOWLES AND HERBERT GINTIS

Princeton University Press: 2011. 288 pp. $\$ 35, £ 24.95$ Around the same time, geneticists Luigi Luca Cavalli-Sforza and Marcus Feldman introduced the methods of population genetics to the study of cultural evolution.

Bowles and Gintis seek to explain, as did Darwin, why humans have moral sentiments. Most people care about the welfare of others, even beyond kin and close associates. Economists use experimental games to test the assumption that humans are selfinterested actors, and often find it wanting. Many participants show fairness and cooperation, punishing those who behave selfishly. The term 'strong reciprocity' is used to describe the tendency of people to sacrifice their own payoffs by rewarding or punishing others. Even in anonymous groups in the laboratory, strong reciprocity deters free-riding and sustains cooperation. Bowles, Gintis and their colleagues have used games to probe the moral sentiments of a range of societies in Africa, Indonesia and South America. They find that cultures vary greatly in their social preferences. Nowhere do individuals, on average, act with complete self-interest, but how much they will pay to punish others varies. Moreover, the groups' experimental behaviour correlates with that in the real world. Societies living in hamlets within tropical forests who cooperate little in real life also show little regard for the welfare of others in the games. By contrast, an Indonesian society of whale hunters rates highest on strong reciprocity.

The lack of completely selfish societies and the variability in strong reciprocity across different societies implies that the evolution of cooperation involves both genes and culture. The question is how the two systems interacted during our evolutionary history.

A Cooperative Species attacks this problem theoretically and empirically. After introducing the mathematical models that have been used to explain the evolution of social behaviour in humans and other animals, the authors caution that some are implausible. For example, game theorists have discovered that these games have many solutions and different groups might choose different rules to govern and reward behaviour by negotiation among self-interested parties. Bowles and Gintis point out that the solution adopted by a group emerges as a result of historical processes and is hard to change by negotiation or persuasion. 
To illustrate how cooperative behaviour may have arisen, the authors build simple computer simulations of Pleistocene human societies. Plausibly, they propose that the crucial step towards human social systems was the evolution of a cooperative unit that was big enough to insure against the risks involved in hunting large game, comprising around 32 adults plus juveniles and the elderly. Such bands, the authors argue, would have a modest amount of genetic variation between them. So, even if violent conflict between bands was common, group selection could not favour costly altruistic acts.

Selection among groups for cooperation gains traction only where it exceeds that for selfishness within a group. Social institutions can reduce the advantages of selfish behaviour. For example, modern hunter-gatherers typically share their resources. More successful providers are 'taxed' in food to support the collective. This sharing limits variation in reproductive success within groups, imposing selection on groups for individually costly acts of cooperation.

The authors' modelling shows that other institutions - such as ostracism, coordinating punishment of defectors and restricting altruism to the local genetically related group - can have the same effects. They suggest that selection can favour emotions such as shame or guilt that internalize social norms and benefit the group but cost the individual.

Although A Cooperative Species is broadly representative of the gene-culture co-evolutionary approach to human cooperation, I beg to differ on some points. In my view, the critical late-Pleistocene groups in which altruism should be explained are the larger tribes composed of many bands. These have the crucial feature of substantial cooperation between genetically unrelated individuals, on which the evolution of complex societies is based. Although bands do sometimes have violent conflicts, intratribal relations are usually more peaceful than intertribal ones. In my opinion, the authors also accept too high a value for the genetic differences between neighbouring populations in their simulations. However, such a discussion illustrates the book's strength. By presenting clear models that are tied tightly to empirically derived parameters, Bowles and Gintis encourage much-needed debate on the origins of human cooperation.

Peter Richerson is professor emeritus in the Department of Environmental Science and Policy, University of California,

Davis, California 95616, USA.

e-mail:pjricherson@ucdavis.edu

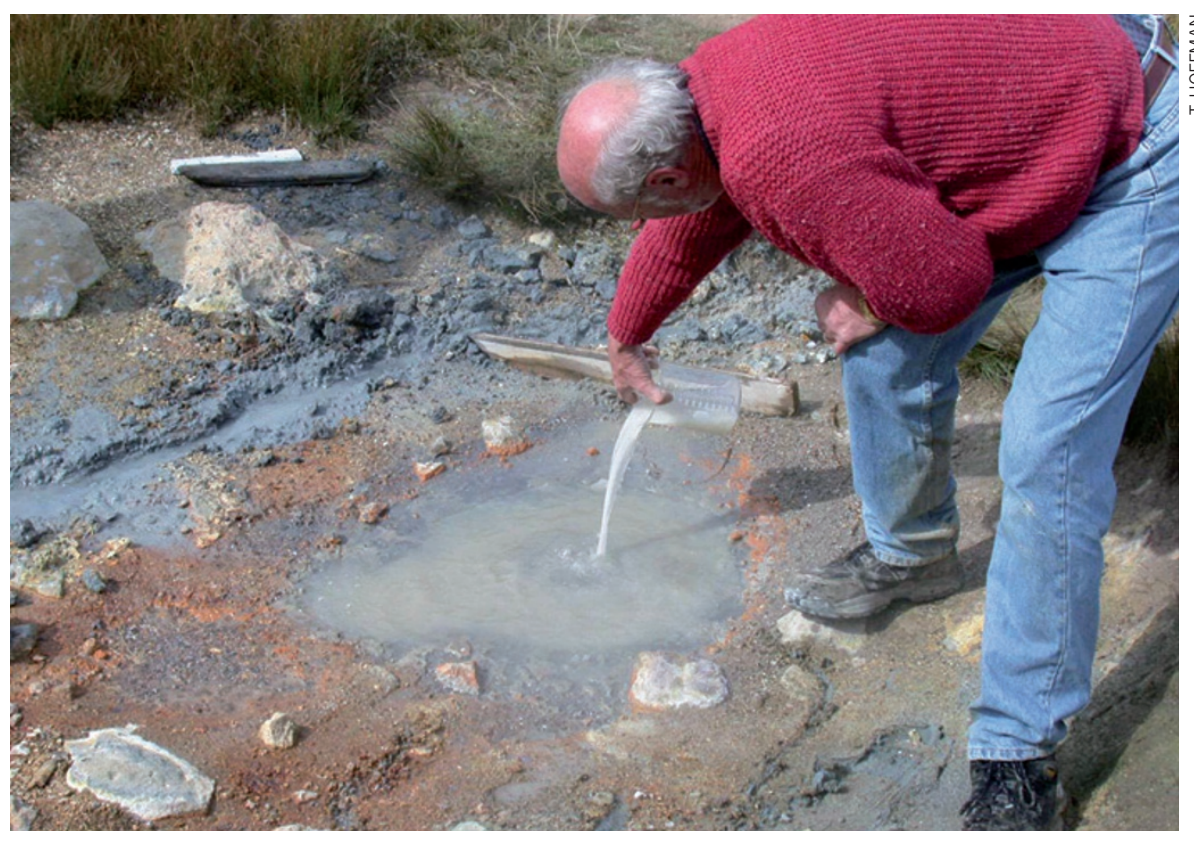

Biochemist David Deamer poured chemicals into a hot pool in 2005 to see if primitive cells would form.

ASTROBIOLOGY

\section{Life's beginnings}

\section{Robert Shapiro on a reminder that laboratory experiments don't always translate to nature.}

I n June 2005, an group of international scientists clustered around a small, near-boiling pool in a volcanic region of Siberia. Biochemist David Deamer took a sample of the waters, then added to the pool a concoction of organic compounds that probably existed 4 billion years ago on the early Earth. One was a fatty acid, a component of soap, which his laboratory studies suggested had a significant role in the origin of life.

Over several days, Deamer took many more samples. He wished to see whether the chemical assembly process that he had observed in his laboratory, which eventually produced complex 'protocell' structures, could also take place in a natural setting. The answer was a resounding no. The clays and metal ions present in the Siberian pool blocked the chemical interactions.

This experiment was a reality check, explains Deamer in First Life. He proposes in the book that the complex molecules that led to life developed not in 'warm little ponds', but in tiny droplets bound by fatty acids. Although his account lacks the tales of personality and conflict that enliven other works in astrobiology, Deamer's $\checkmark$ NATURE.COM For more on the RNA-world hypothesis, see: go.nature.com/kuolyb

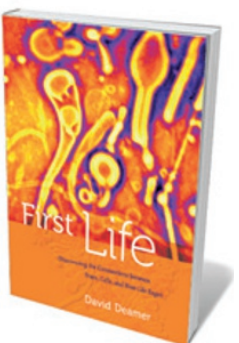

First Life:

Discovering the Connections

between Stars, Cells, and How Life Began DAVID DEAMER

University of California Press: 2011. 288 pp. $\$ 28.95$, £19.95 demonstration that we cannot translate lab results to natural settings is valuable.

Because we can get reactions to work in the controlled conditions of a laboratory, he cautions, it does not follow that similar ones occurred on prebiotic Earth. We might overlook something that becomes apparent when we try to reproduce the reactions in a natural setting. This provocative insight explains why the origin-of-life field has been short on progress over the past half century, whereas molecular biology has flourished.

Today, the simplest living cells depend on molecules that are far more intricate than those that have been isolated from sources unrelated to life (abiotic), such as meteorites. The most noteworthy chemical substances in life are functioning polymers - large molecules made of smaller units called monomers, connected in a specific order. The nucleic acids RNA and DNA, carriers of 